\title{
The Renal Functional Defect of Postobstructive Nephropathy
}

\author{
THE EFFECTS OF BILATERAL URETERAL \\ OBSTRUCTION IN THE RAT
}

\author{
JOHN R. JAENIKE \\ From the Department of Medicine, University of Rochester School of Medicine \\ and Dentistry, Rochester, New York 14642
}

\begin{abstract}
A в S T R A C T This study was designed to examine the pathogenesis of the excretory defect produced by bilateral ureteral obstruction in the rat. After release of obstruction of $24 \mathrm{hr}$ duration glomerular filtration rate was reduced to $20 \%$ of normal. Free flow proximal tubular pressure was normal, excluding residual obstruction as a cause of depressed filtration, and indicating that an intrarenal hemodynamic abnormality was primarily responsible for the excretory defect. Total renal blood flow and cortical distribution of flow were normal. Clearance and micropuncture studies indicated the presence of marked heterogeneity of nephron function with residual excretory function residing primarily in vasodilated nephrons in which decreased postglomerular arteriolar resistance effected a reduction in glomerular filtration pressure. Heterogeneity of nephron function was evidenced by a wide scatter of values for single nephron filtration rate and from direct intratubular injection of dye which revealed that at least $28 \%$ of surface nephrons were either nonfiltering or had filtration rates too low to measure. The observed decrease in Hippuran extraction and increased ratio of Hippuran to inulin clearance ratio is characteristic of the vasodilated kidney. Further evidence of the vasodilated nature of residual functioning nephrons was demonstrated by the failure of intrarenal papaverine infusion to increase filtration rate in this lesion.
\end{abstract}

The hemodynamic defect produced by bilateral obstruction is contrasted with that seen after release of unilateral ureteral ligation in which depression of filtration rate appears to result primarily from preglomerular vasoconstriction. This difference raises the possibility that a vasodilating substance accumulates during total suppression of renal excretory function.

Dr. Jaenike is a recipient of U. S. Public Health Service Career Development Award 2-K3-HE-4526.

Received for publication 4 February 1972 and in revised form 10 August 1972.
Diuresis and natriuresis were constant features of the postobstructive lesion. The present data support previously published studies which localize the defect in sodium transport to the distal nephron and indicate that this defect is a consequence of increased intraluminal pressure and tubular dilatation induced by bilateral ureteral ligation.

\section{INTRODUCTION}

Knowledge of factors which control glomerular filtration is central to our understanding of the basic excretory function of the kidney. It has been shown that a number of experimentally induced (1-3) and clinically occurring (4) varieties of acute renal failure are characterized by alterations in intrarenal hemodynamics which effect marked changes in renal excretory function. Further understanding of pathogenetic factors in these abnormal states will not only enhance knowledge of basic disease mechanisms but will also provide insight into fundamental renal regulatory mechanisms. For this purpose it is desirable to find relatively simple and reproducible models of renal dysfunction which result primarily from aberrations in intrarenal hemodynamic regulation. Previous studies from this laboratory have shown that after release of unilateral ureteral ligation in the rat the obstructed kidney shows a profound reduction in filtration rate which is attributable to renal vasoconstriction, affecting primarily the preglomerular vasculature (5). The present study was designed to investigate the renal functional defect produced by complete bilateral ureteral obstruction in the rat. We had previously noted a striking difference in the appearance of surface tubules of the obstructed kidney depending on whether one or both ureters had been ligated. After unilateral ligation, the tubules were collapsed and showed an intraluminal hydrostatic pressure which was significantly depressed below normal free flow proximal tubular pressure (5). In 
contrast, after bilateral ligation, tubules were dilated and the pressure, before release of obstruction, was elevated (6). These observation indicated that the intrarenal hemodynamic response to bilateral ureteral obstruction differed fundamentally from that of unilateral obstruction.

Diuresis and natriuresis are well recognized features of postobstructive nephropathy in man (7). These also occur after release of bilateral obstruction in the rat (6) and the nature of the defect in sodium transport in this lesion has recently been reported on in detail (8). While certain of the present data also bear on the mechanism of natriuresis in this lesion the major objective of the present study is an examination of the intrarenal hemodynamic events which characterize postobstructive nephropathy and of the manner in which these alterations impair excretory function in this lesion.

\section{METHODS}

Experiments were performed on female Wistar rats, Rochester strain, weighing from 180 to $250 \mathrm{~g}$. Ureteral ligation was performed through a small midline incision, using light pentobarbital anesthesia. Except where otherwise noted, rats were then allowed free access to water, without food, until the time of study, $24 \mathrm{hr}$ later. Animals were then anesthetized with intraperitoneal sodium pentobarbital and placed on a heated platform. Inulin was administered into the jugular vein with a constant infusion pump. Blood pressure was monitored with a pressure transducer (Statham Instruments, Inc., Oxnard, Calif.) attached to a catheter in the carotid artery. The trachea was cannulated. Urine was collected in a weighed $2 \mathrm{ml}$ centrifuge tube from a PE 50 catheter placed in the left ureter. The right ureter remained ligated throughout the experiment. During operative preparation the rat was given $2 \mathrm{ml}$ isotonic $\mathrm{NaCl}$ intravenously, including $100 \mathrm{mg}$ inulin as a priming dose, and inulin in isotonic $\mathrm{NaCl}(20 \mathrm{mg} / \mathrm{ml})$ was thereafter infused at a rate of $0.028 \mathrm{ml} / \mathrm{min}$. Clearance measurements were started $40-45$ min after ureteral catheterization. Periods were of 10-15 min duration. Three periods were taken except in experiments involving the infusion of vasoactive drugs in which two control periods were followed by two to three periods during drug administration.

The effects of the vasoconstrictor agents, norepinephrine and angiotensin, were studied by the intravenous infusion of equipressor amounts in two groups of rats. Norepinephrine was infused at a rate of $0.2 \mu \mathrm{g} / \mathrm{min}$ and angiotensin at 0.026 $\mu \mathrm{g} / \mathrm{min}$. Each drug was infused until blood pressure was stable, usually 10-15 min, after which further clearance measurements were made. Renal vasodilatation was produced by the infusion of papverine $(0.13 \mathrm{mg} / \mathrm{min})$ directly into the renal artery (5). Clearance measurements were initiated 10-15 $\mathrm{min}$ after starting papaverine infusion.

Micropuncture studies were performed by methods previously described (3). Single nephron glomerular filtration rate $\left(\right.$ GFR) ${ }^{1}$ was measured with inulin- ${ }^{-14} \mathrm{C}$ (New England Nuclear Corp., Boston, Mass.). Surface tubules, all of which were dilated and relatively uniform in appearance, were punctured randomly for measurement of GFR. In some tubules the oil block did not move distally over a period as

\footnotetext{
${ }^{1}$ Abbreviations used in this paper: C, clearance; GFR, glomerular filtration rate; $\mathrm{PAH}, p$-aminohippurate; $\mathrm{SNG}$ FR, single nephron glomerular filtration rate.
}

long as $60 \mathrm{sec}$, precluding fluid collection and measurement of inulin clearance in those nephrons. In order to evaluate this more fully, 346 proximal tubules in six postobstructive rats were punctured and a small volume of Lissamine green was rapidly injected into the lumen. The pipette was immediately withdrawn and the time required for the dye to be washed downstream from the point of injection was recorded with a stopwatch. In normal rats and in the majority of tubules in postobstructive rats this occurs immediately or within a few seconds, and moderate reductions in tubular fluid flow rate could not be ascertained by this method. The technique did however clearly distinguish a population of nephrons in which flow was markedly reduced or virtually absent and this permitted a quantification of surface tubules which were essentially nonfunctional.

Renal blood flow was measured by the whole blood clearance of Hippuran- ${ }^{131}$ I (Mallinckrodt Chemical Works, St. Louis, Mo.), corrected for renal extraction by sampling from a catheter previously placed in the renal vein via the left ovarian vein (9).

Distribution of renal blood flow was studied with the use of $15-\mu$ microspheres, labeled with strontium-85 (3M Co. St. Paul, Minn.). Microspheres were suspended in 15\% glucose containing a small amount of Tween-80 (polyoxyethylene sorbitan monooleate). They were sonicated for 5 min immediately before injection. Microscopic examination indicated complete dispersion of the microspheres. Rapid injection of $0.1 \mathrm{mg}$ microspheres suspended in $0.25 \mathrm{ml}$ fluid was performed through a PE 10 catheter threaded into the aorta via the left iliac artery so that the tip lay cephalad to the orifices of both renal arteries. At the end of the experiment $0.8 \mathrm{ml} \mathrm{India} \mathrm{ink} \mathrm{was} \mathrm{injected} \mathrm{through} \mathrm{the} \mathrm{cath-}$ eter, the kidneys were removed and fixed in formalin overnight, to facilitate cutting. They were then hand cut, under the dissecting microscope, for radioactive counting. For this purpose, $10-12$ pieces of kidney, measuring approximately $3 \times 3 \times 5 \mathrm{~mm}$ and including the entire cortex and a portion of outer medulla were cut. Each piece was then cut into five approximately equal slices, from superficial cortex to outer medulla. The corresponding slices from each piece of kidney were pooled, weighed, and assayed in an automatic gamma spectrometer. They were then digested in $0.5 \mathrm{ml}$ of $20 \% \mathrm{HCl}$ at $45^{\circ} \mathrm{C}$ for $18-24 \mathrm{hr}$. After digestion the specimens were thoroughly mixed and duplicate $20-\mu 1$ samples were pipetted from each, placed on a glass slide with a shallow central well, and sealed with a cover slip. The number of glomeruli in each sample was then counted under the microscope. Glomeruli were readily recognizable by their characteristic morphology and heavy staining with India ink.

Sodium was determined by flame photometry. Whole kidney GFR was determined from the clearance of inulin, determined chemically (10). Standard statistical methods were used. Student's $t$ test was used to compare group means.

\section{RESULTS}

Microscopic appearance. Examination of the kidney in situ $24 \mathrm{hr}$ after bilateral ureteral ligation revealed that the surface tubules were markely dilated with clear, translucent fluid. The kidney was swollen and tense. Mean hydrostatic pressure within proximal tubules before release of obstruction was $29.0 \mathrm{~mm} \mathrm{Hg}$ ( $\mathrm{sE} 0.2$ ). These findings are in marked contrast to those previously reported after unilateral ureteral ligation (5), in 
TABLE I

Clearance Data in Normal and Postobstructive Rats

\begin{tabular}{lcccc}
\hline & $\begin{array}{c}\text { No. } \\
\text { rats }\end{array}$ & Inulin clearance & Urine flow & $\mathrm{C}_{\mathrm{Na}} / \mathrm{C}_{\text {in }}$ \\
\hline & & $m l / m$ min per $100 \mathrm{~g}$ & $\mu l / m i n$ & \\
$\begin{array}{l}\text { Normal } \\
\begin{array}{c}\text { Post- } \\
\text { obstruction }\end{array}\end{array}$ & 10 & $0.510 \pm 0.026^{*}$ & $5.5 \pm 0.6$ & $0.006 \pm 0.001$ \\
\hline
\end{tabular}

* Mean \pm SEM.

All values are for one kidney.

which the surface tubules were collapsed and opaque, with a mean intratubular pressure of $8.7 \mathrm{~mm} \mathrm{Hg}$ ( $\mathrm{sE}$ 0.8 ).

Glomerular filtration rate, urine flow, and sodium excretion. Clearance data from the left kidneys of 30 rats studied after release of obstruction, $24 \mathrm{hr}$ after bilateral ureteral ligation, are summarized in Table I. Comparable data from 10 normal rats are shown. A characteristic set of functional changes was noted after release of obstruction. Glomerular filtration rate (GFR) was markedly depressed to approximately $20 \%$ of normal. Fractional sodium excretion was increased to $14 \%$ of the filtered load and all animals showed a high urine flow rate.

Single nephron GFR (SNGFR) was measured in 52 tubules from 10 postobstructive rats. Mean values of SNGFR and tubular fluid to plasma inulin concentration ratios are shown in Table II. Mean SNGFR was significantly reduced $(P<0.01)$, but the reduction was not proportional to that in whole kidney GFR. Whereas whole kidney GFR was reduced to $18 \%$ of normal SNGFR was $34 \%$ of normal. Tubules in which the oil column failed to move distally past the pipette tip were necessarily excluded from the computation of mean values for SNGFR. Lissamine green injection revealed that in 57 of 346 tubules (16\%) there was no detectable downstream movement of dye for $1 \mathrm{~min}$ after injection, indicating virtually complete absence of function in these nephrons. In addition 40 tubules (12\%) showed unequivocal marked reduction in flow rate, the dye requiring more than $10 \mathrm{sec}$ to pass distal to the point of injection. It is not certain whether SNGFR could have been recorded in the latter group of tubules, but this appears unlikely since this represents a distinct subpopulation of nephrons which occurred at a lesser frequency than that observed for the lower range of values of SNGFR (4-8 $\mathrm{nl} / \mathrm{min})$, as shown in Fig. 1 . It therefore appears that at least $28 \%$ of surface nephrons had GFR values below those which could be measured by standard micropuncture methodology, indicating that the mean SNGFR is not truly representative of the composite function of surface nephrons.
TABLE II

Single Nephron Clearances in Normal and Postobstructive Rats

\begin{tabular}{lccccc}
\hline & $\begin{array}{c}\text { No. } \\
\text { rats }\end{array}$ & $\begin{array}{c}\text { No. } \\
\text { tu- } \\
\text { bules }\end{array}$ & SNGFR & $\begin{array}{c}\text { Whole } \\
\text { kidney } \\
\text { GFR }\end{array}$ & $(\mathrm{TF} / \mathrm{P})_{\mathrm{IN}}$ \\
\hline & 11 & 67 & $27.3 \pm 1.4^{*}$ & 0.810 & $2.00 \pm 0.07$ \\
$\begin{array}{l}\text { Normal } \\
\begin{array}{l}\text { Post- } \\
\text { obstruction }\end{array}\end{array}$ & 10 & 52 & $9.2 \pm 0.7$ & 0.149 & $2.20 \pm 0.08$ \\
\hline
\end{tabular}

* Mean \pm SEM.

Mean value of whole kidney GFR was weighted according to number of single nephron GFR determinations per rat.

$(\mathrm{TF} / \mathrm{P})_{\text {IN }}$, proximal tubular fluid to plasma inulin concentration ratio.

The tubular fluid to plasma inulin concentration ratio in postobstructive rats was not significantly different from normal. This finding indicates that the natriuresis was not associated with a detectable proximal tubular defect in sodium transport.

Renal hemodynamics. Renal blood flow was measured by the whole blood clearance of Hippuran- ${ }^{18} I$, corrected for extraction. The results are summarized in Table III. Renal blood flow in postobstructive rats was not significantly different from that observed in normal animals, despite the marked reduction of GFR in the former group. The whole blood extraction of Hippuran and the filtration fraction were markedly and consistently reduced in postobstructive rats.

The cortical distribution of blood flow was measured with radioactive microspheres in order to determine whether redistribution of flow could account for the observed discrepancy between SNGFR and whole kidney GFR and whether this might be a factor in the natriuresis and diuresis. The distribution of radioactivity was related to the distribution of glomeruli in order to provide an index of relative rates of glomerular perfusion at various levels of the renal cortex. As shown in Table IV, in normal rats the outermost cortical zone was the most heavily perfused, over $50 \%$ of total flow

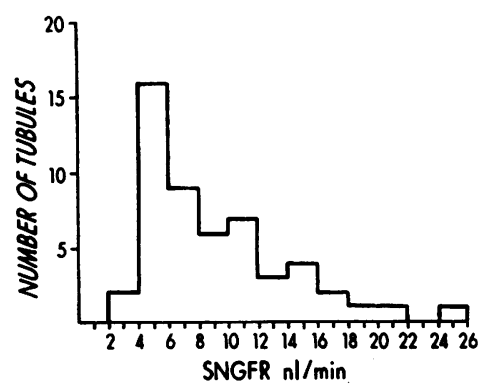

Figure 1 The distribution of single nephron glomerular filtration rates (SNGFR) in postobstructive rats.

Postobstructive Nephropathy in the Rat 
TABLE III

Renal Blood Flow in Normal and Postobstructive Rats

\begin{tabular}{lccccc}
\hline & $\begin{array}{c}\text { No. } \\
\text { rats }\end{array}$ & Inulin clearance & $\begin{array}{c}\text { Renal } \\
\text { blood flow }\end{array}$ & Cin/RBF & $\begin{array}{c}\text { Hippuran } \\
\text { extraction }\end{array}$ \\
\hline & & $m l / m$ in per $100 \mathrm{~g}$ & $m l / m$ m per $100 \mathrm{~g}$ & & \\
Normal & 10 & $0.502 \pm 0.028^{*}$ & $2.76 \pm 0.10$ & $0.182 \pm 0.007$ & $0.43 \pm 0.02$ \\
Postobstructive & 18 & $0.104 \pm 0.010$ & $2.62 \pm 0.24$ & $0.044 \pm 0.004$ & $0.14 \pm 0.02$ \\
\hline
\end{tabular}

* Mean \pm sem.

All values are for one kidney.

$\mathrm{RBF}$, renal blood flow:

perfusing the outermost $25 \%$ of glomeruli. A middle zone, comprising $41 \%$ of glomeruli, received $31 \%$ of total flow, and the innermost zones, containing $34 \%$ of glomeruli, received only $14 \%$ of the total blood flow. The distribution of radioactivity in eight postobstructive rats was not significantly different from the normal. These data have been plotted diagrammatically (Fig. 2) by factoring the fractional distribution of radioactivity by the fractional number of glomeruli in each zone in order to depict the relative rates of glomerular perfusion throughout the renal cortex. The functional defects observed after release of obstruction do not appear to be associated with or attributable to an abnormal distribution of renal blood flow.

The effect of various vasoactive agents was studied in the postobstructive state. Renal vasodilatation was induced by the infusion of papverine $(0.13 \mathrm{mg} / \mathrm{min})$ into the left renal artery. In nine rats mean GFR was unaffected by papaverine, averaging $0.09 \mathrm{ml} / \mathrm{min}$ per $100 \mathrm{~g}$ before and during infusion. This is in striking contrast to the effect of papaverine after release of

TABLE IV

The Cortical Distribution of Radioactive Microspheres in Normal and Postobstructive Rats

\begin{tabular}{llccc}
\hline & \multicolumn{3}{c}{ Fractional distribution } \\
\cline { 3 - 4 } & & & & $\begin{array}{c}\text { Ratio } \\
\text { cpm/G }\end{array}$ \\
\hline Normal & Slice & cpm & G & $0.21 \pm 0.09^{*}$ \\
$\quad(\mathrm{n}=14)$ & 2 & 0.542 & 0.250 & $2.21 \pm 0.05$ \\
& 3 & 0.308 & 0.405 & $0.77 \pm 0.05$ \\
& $4+5$ & 0.042 & 0.089 & $0.48 \pm 0.06$ \\
Postobstruction & 1 & 0.551 & 0.279 & $2.06 \pm 0.13$ \\
$\quad(\mathrm{n}=8)$ & 2 & 0.319 & 0.373 & $0.86 \pm 0.03$ \\
& 3 & 0.094 & 0.263 & $0.36 \pm 0.03$ \\
& $4+5$ & 0.038 & 0.087 & $0.50 \pm 0.11$ \\
\hline
\end{tabular}

* SEM.

cpm, counts per minute corrected for background; G, glomeruli. unilateral obstruction, in which mean GFR rose from 0.019 to $0.167 \mathrm{ml} / \mathrm{min}$ per $100 \mathrm{~g}$ (5).

The effect of vasoconstrictor agents was also studied by administering equipressor amounts of angiotensin and norepinephrine intravenously. The results are summarized in Table V. Norepinephrine induced a small but significant rise $(P<0.05)$ in GFR whereas angiotensin reduced GFR by nearly $50 \%$. Neither agent exerted a significant effect on fractional sodium excretion.

Proximal tubular pressure. Despite high urine flow rate proximal tubular pressure was normal in postobstructive rats. Mean tubular pressure was 13.8 ( $\mathrm{sE}$ 0.3 ) $\mathrm{mm} \mathrm{Hg}$, compared to 13.2 (SE 0.2) $\mathrm{mm} \mathrm{Hg}$ in normal rats $(P>0.10)$. The presence of normal intratubular pressure during diuresis excludes residual tubular obstruction as a causative factor in the reduction of GFR.

Effect of hydration on the postobstructive lesion. The possibility that the functional defect associated with this lesion were influenced by volume expansion in the functionally anephric rat was investigated. In seven rats water was withheld during the $24 \mathrm{hr}$ between ureteral ligation and release of obstruction. All animals lost

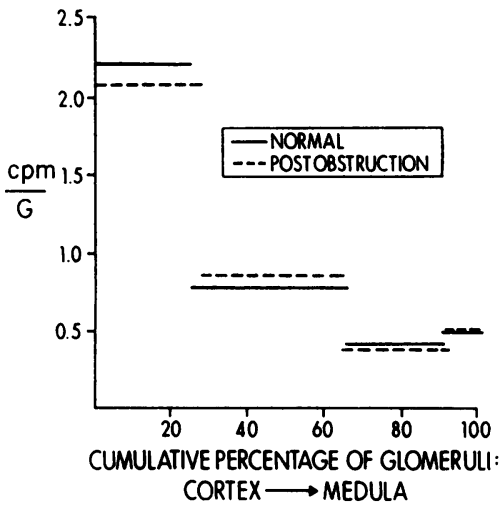

FIGURE 2 The relative rates of glomerular perfusion (cpm/ $G$ ) at various levels of the renal cortex, determined by the distribution of radioactive microspheres, in normal and postobstructive rats. 


\section{TABLE V}

Effects of Angiotensin and Norepinephrine Infusions in Postobstructive Rats

\begin{tabular}{|c|c|c|c|c|c|}
\hline & & Inulin clearance & Urine flow & $\mathrm{C}_{\mathrm{Na}} / \mathrm{C}_{\mathrm{in}}$ & MAP \\
\hline & & $m l_{/}^{\prime}$ min per $100 \mathrm{~g}$ & $\mu l / m i n$ & & \\
\hline \multirow[t]{2}{*}{ Norepinephrine $(n=7)$} & Control & $0.093 \pm 0.008^{*}$ & $39 \pm 6$ & $0.13 \pm 0.01$ & $101 \pm 5$ \\
\hline & Pressor & $0.115 \pm 0.014$ & $52 \pm 10$ & $0.13 \pm 0.01$ & $125 \pm 3$ \\
\hline \multirow[t]{2}{*}{ Angiotensin $(n=6)$} & Control & $0.099 \pm 0.032$ & $40 \pm 10$ & $0.14 \pm 0.02$ & $104 \pm 9$ \\
\hline & Pressor & $0.054 \pm 0.017$ & $17 \pm 4$ & $0.13 \pm 0.02$ & $124 \pm 8$ \\
\hline
\end{tabular}

* Mean \pm SEM.

MAP, mean arterial blood pressure.

weight during this period, a mean of $8 \mathrm{~g}$. The fractional sodium excretion in this group was 0.153 , not different from that observed in rats allowed free access to water (Table I). Other renal functions were also unaltered by water deprivation. GFR averaged 0.092 $\mathrm{ml} / \mathrm{min}$ per $100 \mathrm{~g}$ and renal blood flow $2.80 \mathrm{ml} / \mathrm{min}$ per $100 \mathrm{~g}$, values comparable to those in water-drinking rats (Tables I and III).

Effect of urea diuresis. It was postulated that solute diuresis due to previously retained urea might be responsible for postobstructive natriuresis. Urea was infused into normal rats and rats with previous unilateral ureteral ligation to achieve plasma urea nitrogen concentrations comparable to those found $24 \mathrm{hr}$ after bilateral ligation (25-41 mm). As shown in Table VI only slight degrees of natriuresis resulted. Fractional sodium excretion did not exceed 0.024 in normal rats or 0.015 in unilaterally obstructed animals. The marked natriuresis seen after release of bilateral obstruction does not appear to be attributable primarily to solute diuresis.

\section{DISCUSSION}

A characteristic set of renal functional defects has been observed in the rat after release of bilateral ureteral ob- struction, namely, diuresis, natriuresis, and impairment of glomerular filtration. Identical findings have been described in patients after relief of urinary obstruction $(7,11-14)$. While a number of recent studies have been directed to the renal functional changes associated with unilateral obstruction $(15,16)$ and acute $(17,18)$ and chronic (18) elevation of ureteral pressure, data obtained after release of total obstruction have been sparse and related primarily to the defects in sodium and water excretion in this lesion (8). The impairment of glomerular filtration after release of bilateral obstruction has remained unexplored and the present study is directed primarily to determining the pathogenesis of this excretory defect.

The present data indicate that impairment of filtration rate in the postobstructive state results from an intrarenal hemodynamic abnormality. Total renal blood flow and cortical distribution of flow are normal, despite the presence of marked reduction of GFR. The concurrence of normal blood flow with reduced GFR may be present when there is intratubular obstruction to flow (3), but measurements of free flow proximal tubular pressure in the postobstructive state, in the presence of high rates of urine flow, reveal no evidence of tubular obstruction. It is therefore concluded that a hemodynamically deter-

\section{TABLE VI}

The Effect of Urea Infusion on Fractional Sodium Excretion in Normal Rats and after Release of Unilateral Ureteral Obstruction

\begin{tabular}{|c|c|c|c|c|c|c|}
\hline & \multirow[b]{2}{*}{$\begin{array}{l}\text { No. } \\
\text { rats }\end{array}$} & \multirow[b]{2}{*}{$\begin{array}{c}\text { Inulin } \\
\text { clearance }\end{array}$} & \multirow[b]{2}{*}{$\begin{array}{l}\text { Urine } \\
\text { flow }\end{array}$} & \multicolumn{2}{|c|}{$\mathrm{C}_{\mathrm{Na}} / \mathrm{C}_{\mathrm{in}}$} & \multirow[b]{2}{*}{$\begin{array}{c}\text { Plasma } \\
\text { urea }\end{array}$} \\
\hline & & & & Mean & Range & \\
\hline & & $\begin{array}{l}\mathrm{ml} / \mathrm{min} \\
\text { per } 100 \mathrm{~g}\end{array}$ & $\mu l / \min$ & & & $m \mathrm{M}$ \\
\hline Normal & 2 & $1.29^{*}$ & $72.5^{*}$ & 0.022 & $0.020-0.024$ & 27.5 \\
\hline Unilateral obstruction & 4 & $0.26 \ddagger$ & $7.5 \ddagger$ & 0.008 & $0.004-0.015$ & 35.4 \\
\hline
\end{tabular}

Urea was infused intravenously as follows : 4.7 mmoles in $2.0 \mathrm{ml} 0.075 \mathrm{M} \mathrm{NaCl}$ as a prime, followed by infusion of 600 $\mathrm{mM}$ urea in isotonic $\mathrm{NaCl}$ at $0.05 \mathrm{ml} / \mathrm{min}$.

* Both kidneys.

$\ddagger$ Left kidney only. 
mined reduction of effective glomerular filtration pressure is present in this lesion. According to present concepts of the dynamics of glomerular filtration, filtration pressure is a function of renal perfusion pressure and of segmental vascular resistances within the kidney. Although the equations for the calculation of segmental resistances (19) may not be formally applicable to a kidney in which there is marked heterogeneity of nephron function, the observed relationship between renal blood flow and GFR permits certain qualitative conclusions. The presence of a normal total renal blood flow, with normal perfusion pressure, indicates that total renal vascular resistance was normal. This excludes afferent glomerular arteriolar constriction as the sole or primary hemodynamic defect in this lesion since this would effect an increase in total resistance and a consequent reduction in renal blood flow. Impairment of glomerular filtration pressure in this lesion can be explained only by some combination of afferent vasoconstriction and efferent arteriolar dilatation. This might be a homogenous effect, with all nephrons similarly involved, or it might reflect the coexistence of two populations of nephrons, one of which is vasoconstricted, poorly perfused and essentially nonfunctional, and the other manifesting predominate efferent vasodilatation with resultant hyperperfusion. Several lines of evidence suggest that the latter pattern exists in the postobstructive kidney and that the vasodilated nephrons make the major contribution to excretory function in this lesion. First, measurements of SNGFR show marked heterogeneity of glomerular function, and intratubular injection of dye reveals that a substantial fraction of surface nephrons are essentially nonfiltering, clearly indicating a wide divergence of hemodynamic function among various nephrons. Secondly it has been shown that renal vasodilatation does not enhance GFR in this lesion, in marked contrast to its effect after release of unilateral ureteral obstruction, a lesion in which the defect in GFR appears to result primarily from afferent glomerular arteriolar constriction (5). This suggests that the final urine after release of bilateral obstruction is the product primarily of vasodilated nephrons, unresponsive to further, pharmacologically induced, vasodilatation. Further support of the conclusion that effective excretory function resides mainly in vasodilated nephrons is provided by the pattern of excretion of Hippuran and inulin in this lesion. Although the whole blood extraction of Hippuran was markedly reduced, the ratio of clearance of Hippuran $\left(\mathrm{C}_{\mathbf{H}}\right)$ to that of inulin rose from 2.39 (SE 0.11) in control rats to 3.18 (SE 0.18) after obstruction, a significant difference $(P<0.01)$. A number of factors might be invoked to explain the alterations of Hippuran extraction and clearance in this lesion. Either proximal tubular damage, by specifically impairing Hippuran transport, or a relative increase in medullary blood flow, with consequent delivery of a proportionately higher fraction of postglomerular blood to nonsecretory tubular segments (20), would reduce Hippuran extraction. If either were the primary or sole factor responsible for decreased extraction, $\mathrm{C}_{\mathbf{H}} / \mathrm{C}_{\mathbf{i n}}$ should decrease, whereas the converse was found. Furthermore a significant rise in medullary blood fiow appears to be excluded by the normal pattern of microsphere distribution. Some impairment of tubular transport can not be excluded however and might be masked by hemodynamic factors which enhance $C_{\mathbf{H}}$ relative to GFR, as discussed below. Perfusion of nonfiltering nephrons might also contribute to reduced Hippuran extraction in this lesion but this defect would not alter the relationship between inulin and Hippuran clearances. The concurrence of decreased tubular extraction of $p$-aminohippurate $(\mathrm{PAH})$ with an increase in clearance of $\mathrm{PAH}$, relative to GFR, appears to be a characteristic of the rasodilated kidney. The studies of Earley and Friedler (21) show a consistent reduction of PAH extraction and increase of $\mathrm{C}_{\mathrm{P} \cdot \mathrm{AH}} / \mathrm{C}_{\mathrm{in}}$ after renal vasodilatation induced by acetylcholine, bradykinin, and kallidin. In contrast, $\mathrm{C}_{\mathrm{P} \wedge \mathrm{H}} / \mathrm{C}_{\text {in }}$ fell during vasoconstriction produced by angiotensin infusion. Similar results have been obtained by Aukland and Loyning (22) who also showed that PAH extraction rose transiently after acute reduction of renal blood flow induced by renal artery constriction. These data indicate that alterations in the pattern of glomerular perfusion and filtration may directly influence both the extraction of Hippuran and its clearance relative to GFR. Aukland and Loyning (22) have proposed that in the vasodilated kidney tubular transport of PAH is limited by its rate of diffusion from the peritubular capillaries. They postulate that with increased peritubular capillary flow rate the reduction in transit time leads to a diminished extraction of $\mathrm{PAH}$, although net delivery of $\mathrm{PAH}$ to tubules and thus total clearance rate will increase. This pattern of Hippuran and inulin excretion in the postobstructive kidney suggests the presence of hyperperfusion of those nephrons primarily responsible for residual excretory function. Despite apparent vasodilatation SNGFR is reduced (Fig. 2), indicating that effective glomerular filtration pressure is diminished. This combination of functional changes indicates that decreased resistance in the efferent glomerular arterioles is the primary hemodynamic alteration in this population of nephrons. Postglomerular vasodilatation enhances nephron blood flow while effecting a reduction in nephron GFR consequent to its effect on glomerular capillary hydrostatic pressure (19).

SNGFR in surface tubules is reduced proportionately less than whole kidney GFR in the postobstructive rat. Yarger, Aynedjian. and Bank (8) have made similar 
observations and have suggested that function in superficial cortical nephrons is relatively better preserved than in deeper nephrons, inaccessible to standard micropuncture techniques. In the present study however some surface tubules appeared to be nonfunctional. The oil injected to provide tubular blockade failed to move downstream from the site of injection over a period as long as $60 \mathrm{sec}$. This precluded collection of fluid and measurement of GFR in such tubules and they were consequently excluded from the pooled data. As shown in Fig. 1 there was marked heterogeneity of superficial nephron function. Although the largest number of values fall between 4 and $6 \mathrm{nl} / \mathrm{min}$, a reduction in SNGFR proportional to whole kidney GFR, the necessary exclusion of nephrons with a GFR less than $4 \mathrm{nl} / \mathrm{min}$ results in a skewing of the distribution to the right. Proximal tubular injection of Lissamine green indicates that approximately $28 \%$ of surface nephrons were either nonfiltering or had filtration rates too low to measure by conventional micropuncture technique. These findings suggest that the disparity between surface SNGFR and whole kidney GFR may result solely from limitations of methodology and not represent a true difference in distribution of nephron involvement in this lesion. This view is further supported by the observation that microsphere distribution is not altered in the postobstructive kidney, although since this method measures distribution of blood flow rather than filtration rate it does not exclude the possibility that GFR, relative to blood flow, is better maintained in superficial than in deep nephrons. The marked heterogeneity of nephron function in this lesion has been repeatedly observed in other types of experimentally induced acute renal failure (1-3). While this may have been attributable to varying degrees of tubular damage or intratubular obstruction in individual nephrons in those lesions, the present study indicates that an apparently uniform inciting lesion, total obstruction, produces a widely variable hemodynamic response in individual glomeruli. The mechanism by which individual cortical glomeruli manifest widely disparate hemodynamic responses to a uniform stimulus remains unexplained.

It is well established that enhanced sodium excretion occurs after release of urinary obstruction in man (7, 11-14). The clinical reports of postobstructive natriuresis include only patients in whom there is either bilateral obstruction or unilateral obstruction with absence of the contralateral kidney $(7,11-14)$. This phenomenon does not appear to occur after release of unilateral obstruction when the opposite kidney is functionally intact. While it has been suggested that this natriuresis is an appropriate response to increased total body sodium, accumulated during the period of urinary suppression (11), the present study shows that natriuresis occurs despite total ex- clusion of sodium intake during the period of obstruction. Further it has been shown that neither overhydration, resulting from continuing water intake in the presence of anuria, nor solute diuresis, from retained urea, can account for the enhanced rate of sodium excretion. Several additional possible mechanisms exist. First, it has been considered that natriuresis might result primarily from altered peritubular physical forces. Both the low filtration fraction, by producing a relatively low peritubular capillary oncotic pressure, and postglomerular vasodilatation, which might effect a rise in peritubular capillary hydrostatic pressure, would be expected to decrease fractional sodium reabsorption in the proximal tubule $(23,24)$. Two lines of evidence suggest that this is not the primary mechanism in postobstructive natriuresis. Most compelling is the observation that the proximal tubular fluid to plasma inulin concentration ratio is not depressed, indicating that fractional sodium reabsorption is not reduced. This finding is in agreement with the studies of Yarger et al. (8). Furthermore, renal vasoconstriction, induced by angiotensin and manifested by a marked lowering of GFR, which would be expected to decrease peritubular capillary hydrostatic pressure, did not reduce fractional $\mathrm{Na}$ excretion. Equipressor amounts of norepinephrine did not affect GFR or Na excretion. From the work of Earley and Friedler (21) a natriuretic effect from norepinephrine might be expected if the functioning nephrons were vasodilated. However the present study differs from that of Earley and Friedler (21) in several respects, including a less marked rise in blood pressure and evidence for predominant postglomerular vasodilatation in this lesion as opposed to the generalized dilatation produced by acetylcholine. During vasodilatation induced by acetylcholine the elevated systemic pressure is presumably transmitted to the peritubular capillaries whereas in the present study the preglomerular vessels appear to be responsive to vasoconstricting agents. This is evidenced by the response of GFR to angiotensin in this lesion in contrast to the minimal effect on GFR noted in the study of Earley and Friedler (21). The present data indicate that the defect in sodium transport is located in the distal tubule and it is suggested that this may be a direct consequence of the mechanical effects of prolonged ureteral obstruction in the distal nephron. The possibility that some humoral agent, acting to decrease distal sodium transport, might accumulate during total obstruction and account for the subsequent natriuresis has not been excluded.

The mechanism responsible for the intrarenal hemodynamic response to bilateral obstruction remains undetermined. It is of particular interest that this response differs markedly from that seen after unilateral ureteral ligation (5), in which preglomerular vasoconstriction seems to be the predominant defect. As a result, intra- 
tubular pressure is low, tubules are collapsed and postobstructive natriuresis is absent. In contrast, when both ureters are ligated, postglomerular vasodilatation maintains normal renal perfusion, despite an apparent moderate increase in afferent arteriolar resistance. It is not clear why these two types of obstruction produce such strikingly different intrarenal hemodynamic responses. The most apparent difference in the nature of these lesions is that after unilateral obstruction near-normal renal excretory function persis.s, through the function of the contralateral kidney, whereas after bilateral ureteral ligation the animal is functionally anephric. This suggests that a substance with renal vasodilator properties may accumulate during total suppression of excretory function and account for the differing hemodynamic responses in these two lesions. The possibility that such a substance exists, and that it might be operative in this setting and in the hyperperfusion of residual nephrons in chronic renal disease (25), remains unconfirmed.

\section{ACKNOWLEDGMENTS}

I am indebted to Mrs. Bette S. Vandenburg for her excellent technical assistance. I also wish to thank Mr. James G. Hellerman, medical student fellow, for his excellent work in adapting the microsphere method for use in the rat.

This work was supported by U. S. Public Health Service Grant HE 07966.

\section{REFERENCES}

1. Flanigan, W. J., and D. E. Oken. 1965. Renal micropuncture study of the development of anuria in the rat with mercury-induced acute renal failure. J. Clin. Invest. 44: 449 .

2. Oken, D. E., M. L. Arce, and D. R. Wilson. 1966. Glycerol-induced hemoglobinuric acute renal failure in the rat. I. Micropuncture study of the development of oliguria. J. Clin. Invest. $45: 724$.

3. Jaenike, J. R. 1969. Micropuncture study of methemoglobin-induced acute renal failure in the rat. J. Lab. Clin. Med. 73: 459 .

4. Hollenberg, N. K., M. Epstein, S. M. Rosen, R. I. Basch, D. E. Oken, and J. P. Merrill. 1968. Acute oliguric renal failure in man: evidence for preferential renal cortical ischemia. Medicine (Baltimore). 47: 455.

5. Jaenike, J. R. 1970. The renal response to ureteral obstruction: a model for the study of factors which influence glomerular filtration pressure. J. Lab. Clin. Med. 76: 373

6. Jaenike, J. R. 1970. The hemodynamic lesion of postobstructive nephropathy. American Society of Nephrology, Abstracts of the 4th Annual Meeting, Washington, D. C. 37.

7. Bricker, N. S., E. I. Shwayri, J. B. Reardan, D. Kellog, J. P. Merrill, and J. H. Holmes. 1957. An abnormality in renal function resulting from urinary tract obstruction $A m . J . M e d .23: 554$.
8. Yarger, W. E., H. S. Aynedjian, and N. Bank. 1972. A micropuncture study of postobstructive diuresis in the rat. J. Clin. In''est. 51: 625 .

9. Jaenike, J. R. 1967. The renal lesion associated with hemoglobinemia: a study of the pathogenesis of the excretory defect in the rat. J. Clin. Invest. 46: 378.

10. Walser, M., D. G. Davidson, and J. Orloff. 1955. The renal clearance of alkali-stable inulin. J. Clin. Invest. 34: 1520 .

11. Muldowney, F. P., G. J. Duffy, D. G. Kelly, F. A. Duff, C. Harrington, and R. Freaney. 1966. Sodium diuresis after relief of obstructive uropathy. N. Engl. J. Med. 274: 1294

12. Witte, M. H., F. A. Short, and W. Hollander, Jr. 1964. Massive polyuria and natriuresis following relief of urinary tract obstruction. Am. J. Med. 37: 320 .

13. Wilson, B., D. D. Reisman, and C. A. Moyer. 1951. Fluid balance in the urological patient. Disturbances in the renal regulation of the excretion of water and sodium salts following decompression of the urinary bladder. J. Urol. $66: 805$.

14. Luton, E. F., and F. S. Dietrich. 1967. Postobstructive hypersaluresis. J. Urol. 98: 402.

15. Bercovitch, D. D., L. Kasen, I. Blann, and M. F. Levitt. 1971. The postobstructive kidney. Observations on nephron function after the relief of $24 \mathrm{hr}$ of ureteral ligation in the dog. J. Clin. Iniest. 50: 1154.

16. Vaughan, E. D., Jr., E. J. Sorenson, and J. Y. Gillenwater. 1971. Alterations in renal function immediately after release of acute total unilateral ureteral occlusion. Invest. Urol. 8: 450.

17. Suki, W. N., A. G. Guthrie, M. Martinez-Maldonado, and G. Eknoyan. 1971. Effects of ureteral pressure elevation on renal hemodynamics and urine concentration. Am. J. Physiol. 220: 38.

18. Suki, W., G. Eknoyan, F. C. Rector, Jr., and D. W. Seldin. 1966. Patterns of nephron perfusion in acute and chronic hydronephrosis. J. Clin. Inc'est. 45: 122.

19. Gómez, D. M. 1951. Evaluation of renal resistances, with special reference to changes in essential hypertension. J. Clin. Invest. $30: 1143$.

20. Slotkoff, L. M., G. M. Eisner, and L. S. Lilienfield. 1968. Functional separation of renal cortical-medullary circulation: significance of Diodrast extraction. Am. J. Physiol. 214: 935.

21. Earley, L. E., and R. M. Friedler. 1966. The effects of combined renal vasodilatation and pressor agents on renal hemodynamics and the tubular reabsorption of sodium. J. Clin. Invest. 45: 542.

22. Aukland, K., and E. W. L фyning. 1970. Intrarenal blood flow and para-aminohippurate $(\mathrm{PAH})$ extraction. Acta Physiol. Scand. 79: 95.

23. Daugherty, T. M., L. J. Belleau, J. A. Martino, and L. E. Earley. 1968. Interrelationship of physical forces affecting sodium reabsorption in the dog. Am. J. Phy'siol. 215 : 1442 .

24. Spitzer, A., and E. E. Windhager. 1970. Effect of peritubular oncotic pressure changes on proximal tubular fluid reabsorption. Am. J. Phy'siol. 218: 1188.

25. Bricker, N. S.. S. Klahr, and R. E. Rieselbach. 1964 The functional adaptation of the diseased kidney. I. Glomerular filtration rate. J. Clin. Inz'cst. 43: 1915. 